\title{
The Correlation Between Long-Lasting Serum Lactate And Brain Mri Abnormality In Children With Status Epilepticus
}

Prastiya Indra Gunawan ( $\sim$ prastiya-i-g@fk.unair.ac.id )

Universitas Airlangga/Dr Soetomo General Academic Hospital

Riza Noviandi

Universitas Airlangga/Dr Soetomo General Academic Hospital

Sunny Mariana Samosir

Universitas Airlangga/Dr Soetomo General Academic Hospital

\section{Research Article}

Keywords: brain MRI, children, mortality, serum lactate, status epilepticus

Posted Date: January 24th, 2022

DOI: https://doi.org/10.21203/rs.3.rs-1136749/v1

License: (c) (i) This work is licensed under a Creative Commons Attribution 4.0 International License.

Read Full License 


\section{Abstract}

Background: Serum lactate was a biomarker implemented to estimate the prognosis in children suffered from status epilepticus (SE). Brain neuroimaging may demonstrate the site area, range of neuronal impairment and ischemic injury. This study aims to evaluate the correlation between long-lasting serum lactate with brain imaging abnormality reflected on Magnetic Resonance Imaging (MRI) in children suffered from SE.

Method: This prospective analytical research was conducted from June-November 2018 in children with SE. Serum lactate were collected on 24 hours following SE, and brain MRI was carried out within a period of 7-14 days after SE terminated. Abnormal MRI was categorized as Hypoxic Ischemic Encephalopathy (HIE) grade I to III. Statistical analysis with Kruskal Wallis was applied to calculate the details.

Results: Forty two SE subjects were enrolled, $85.7 \%$ boys, with a mean age of 59.29 months and mostly with the diagnosis of encephalitis (47.6\%). Elevated serum lactate levels were found in $71.4 \%$ of patients and about $47.8 \%$ had abnormal MRI images in the form of HIE Grade I. Mean serum lactate level in HIE grade I, II, III was $1.69 \mathrm{mmol} / \mathrm{L}, 3.32 \mathrm{mmol} / \mathrm{L}, 3.48 \mathrm{mmol} / \mathrm{L}$ respectively. Two patients were death and $28.6 \%$ had a life with neurological deficit. There was a significant correlation $(p=0.021)$ between longlasting serum lactate level and abnormal brain MRI.

Conclusion: In children suffered from SE, HIE grade I is the most neuroimaging pattern obtained, and long-lasting serum lactate was considered to correlate with abnormal brain MRI.

\section{Introduction}

Status epilepticus (SE) is a neurological life-threatening condition with high mortality and morbidity in children. It is characterized by recurrent seizures in the absence of return to baseline or prolonged seizure more than 30 minutes. ${ }^{1}$ Therefore, the assessment of prognosis is very important in SE management to avoid over-treatment and long term complications. ${ }^{2,3}$ Appropriate diagnostic and therapeutic actions are expected to reduce mortality and determine a further prognosis. ${ }^{2-4}$ The prevalence of SE in children is approximately to be 18-23 per 100,000 children per year, mostly in neonates up to the age of 5 years, with a mortality of $2-7 \%{ }^{3}$ In Iran and India, there were 43 and 70 cases with SE respectively in a year. 5,6

Brain Magnetic Resonance Imaging (MRI) in SE aims to evaluate structural damage, identify the cause of seizures, and predict the prognosis of patients. ${ }^{7,8}$ Physiological changes reported included cerebral oedema, hyperperfusion, and changes in the blood-brain barrier. An imaging study found that abnormalities of the brain were reported in $20 \%$ with Computed Tomography (CT) scan, while brain MRI abnormalities were seen in $58 \%$ in SE. ${ }^{1}$

There have been many studies on the correlation of biological fluid biomarkers with neurological disorders. ${ }^{9}$ Lactate levels as a biomarker will increase in the first few hours after the seizure. Formerly the seizure has terminated, the lactate production reduces and it will rapidly cleared. Blood lactate quantity 
reflect the degree of brain damage that occurs and are related with poor prognosis in children. ${ }^{10}$ According to Nass, lactate is a vigorous neurological biomarker of generalized seizures with rising levels identified almost $90 \%$ subjects within a half hour following seizure eradication. ${ }^{11}$ Matz stated that serum lactate level that collected within two hours following generalized tonic-clonic seizures was significantly increased. ${ }^{12}$ Calabrese also stated that cerebrospinal fluid and plasma lactate level that were collected within twelve hours following seizure termination were significantly elevated. ${ }^{13}$ This result demonstrated that SE produces a remarkable elevation in lactate level and may considered as an indicator of morbidity and mortality. ${ }^{12}$ Several studies have reported increasing blood lactate levels and imaging abnormalities

in children with HIE. ${ }^{14}$ Otherwise, no study has been conducted to investigate the evidence of long-lasting serum lactate level that obtained within 24 hours following SE that reflected in the pattern of brain imaging in children. This study aimed to investigate the correlation between long-lasting serum lactate levels and abnormal brain MRI that visualized in children suffered from SE.

\section{Methods}

\section{Design and setting}

A prospective study was conducted from June to November 2018 in children with SE in the Emergency and Pediatric Ward of the hospital.

\section{Selection of participants}

The subjects of this study were all children suffered from SE in the research period. The confirmation of SE was based on the standard international classification; patients who suffered from continue seizures or having multiple seizures without recovering consciousness within 30 minutes or more. ${ }^{1,2}$ The subjects were included if they were aged 1 month to 12 years old, a first episode of SE and agreeing to participate in the study. Informed consent was obtained from parent or legal guardian from all subjects. The exclusion criteria in this study were a history of seizure or traumatic brain injury less than 3 weeks before and congenital anomalies of the central nervous system.

\section{Methods}

Seizure patients who met the criteria would have blood drawn for lactate testing and undergo a head MRI examination. Blood samples for lactate measurement was derived from the arterial vessels irrespective of the site of sampling and processed within 15-30 minutes. In order to obtain long-lasting lactate serum level, the examination of lactate in the blood serum was carried out 24 hours post-seizure resolution . Then, the patient underwent an MRI of the head 7-14 days after the seizure (according to the schedule obtained) with Diffusion Tensor Imaging (DTI) sequences and MR Spectroscopy. Brain MRI was performed using GE magnetic resonance spectroscopy and perfusion (MR360 Optima 1.5T GE). MRI results were divided into three types based on the abnormality location of HIE. Grade I is a disorder 
limited to the white matter tract, Grade II is a cortical and subcortical lesion, and Grade III is a lesion in most white matter. ${ }^{15}$

\section{Outcomes}

Outcome evaluation was performed when the patients were discharged. All data were recorded including age, gender, nutritional status, history of other disease, hemoglobin level, leukocyte level, C-Reactive Protein (CRP) level, serum lactate level, interpretation of the head MRI and length of stay in the hospital.

\section{Ethical Approval}

All methods carried out in this study were carried out in accordance with relevant guidelines and regulations. The ethical approval was obtained from the Ethic and Medicolegal Committee at Dr.Soetomo General Academic Hospital Surabaya with the ethics number 246/Panke.KKE/IV/2017.

\section{Data analysis}

All data were calculated using IBM SPSS Statistics 21 software. The relationship between serum lactate levels and brain MRI abnormalities in SE patients was analyzed using Kruskal Wallis test and Spearman test. A two sided $p$-value of $\leq 0.05$ was considered significant.

\section{Results}

Approximately 44 subjects presented with a seizure of more than 30 minutes. Two patients died before head MRI was performed. Forty-two children who appropriate with the criteria underwent serum lactate examination and brain imaging. The baseline clinical characteristic baseline clinical data of the subjects were seen in Table 1.

Table 1. Baseline characteristic of the patients 


\begin{tabular}{|c|c|}
\hline Characteristic & Value \\
\hline Mean age (month), (SD) & $59.29(46.98)$ \\
\hline \multicolumn{2}{|l|}{ Gender, n (\%) } \\
\hline \multirow{2}{*}{$\begin{array}{l}\text { - Boys } \\
\text { - Girls }\end{array}$} & $36(85.7)$ \\
\hline & $6(14.3)$ \\
\hline \multicolumn{2}{|l|}{ Age groups, $\mathrm{n}(\%)$} \\
\hline \multirow{3}{*}{$\begin{array}{l}\text { - } \quad<3 \text { years old } \\
\text { - } 3-<6 \text { years old } \\
\text { - } 6-12 \text { years old }\end{array}$} & $18(42.8)$ \\
\hline & $10(23.8)$ \\
\hline & $14(33.3)$ \\
\hline \multicolumn{2}{|l|}{ Nutritional status, n (\%) } \\
\hline \multirow{3}{*}{$\begin{array}{l}\text { - } \text { malnutrition } \\
\text { - normal } \\
\text { - obesity }\end{array}$} & $12(28.6)$ \\
\hline & $30(71.4)$ \\
\hline & $0(0.0)$ \\
\hline \multicolumn{2}{|l|}{ Diagnosis, n (\%) } \\
\hline \multirow{3}{*}{$\begin{array}{l}\text { - Encephalitis } \\
\text { - Meningoencephalitis } \\
\text { - Epilepsy }\end{array}$} & $20(47.6)$ \\
\hline & $12(28.6)$ \\
\hline & $10(23.8)$ \\
\hline Mean hemoglobin $(\mathrm{g} / \mathrm{dL}),(\mathrm{SD})$ & $11.39(1.24)$ \\
\hline Mean leukocyte (cell/mL), (SD) & $13857.6(7665.98)$ \\
\hline Mean CRP (mg/L), (SD) & $3.31(4.48)$ \\
\hline \multicolumn{2}{|l|}{ Length of stay, n (\%) } \\
\hline \multirow{2}{*}{$\begin{array}{l}\text { - } \quad<7 \text { days } \\
\text { - }>7 \text { days }\end{array}$} & $4(9.5)$ \\
\hline & $38(90.4)$ \\
\hline \multicolumn{2}{|l|}{ Serum lactate level, n (\%) } \\
\hline \multirow{2}{*}{$\begin{array}{l}\text { - } \quad 0.3-1.3(\mathrm{mmol} / \mathrm{L}) \\
\text { - }>1.3(\mathrm{mmol} / \mathrm{L})\end{array}$} & $12(28.6)$ \\
\hline & $30(71.4)$ \\
\hline \multirow{4}{*}{$\begin{array}{l}\text { Outcomes, n (\%) } \\
\text { - Live without neurological deficit } \\
\text { - Live with neurological deficit } \\
\text { - Died }\end{array}$} & \\
\hline & $12(28.6)$ \\
\hline & $28(66.7)$ \\
\hline & $2(4.8)$ \\
\hline
\end{tabular}


MRI finding, $\mathrm{n}(\%)$

- Normal $2(4.8)$

- HIE grade I

- HIE grade II

$20(47.6)$

- HIE grade III

The participants in the study had an age range of 1 month to 12 years, with a mean age of 59.29 months. Most of them were boys (85.7\%). Nutritional status was found to be normal in $30(71.4 \%)$ and the majority of $20(47.6 \%)$ SE cases were encephalitis, whereas the etiology of 10 (28.6\%) was epilepsy. Around 38 (90.4\%) SE cases were treated for more than seven days at the hospital. A favorable outcome at hospital discharge was found in 12 SE cases (28.6\%), whereas 28 SE cases (66.7\%) lived with neurological deficit and 2 (4.8\%) ended fatally.

This study showed that only 2 (4.8\%) SE child had normal brain MRI. The remaining SE children had abnormal MRI in the form of HIE Grade I (47.8\%), HIE Grade II (28.6\%), and HIE Grade III (14.3\%). The macroscopic appearances of the head MRI samples were seen in Figure 1. Normal serum lactate levels are $0.3-1.3 \mathrm{mmol} / \mathrm{L}$; however, $71.4 \%$ of patients with SE showed an elevation of serum lactate quantity.

A comparative assessment was performed between serum lactate levels with the degree of encephalopathy. The degree of encephalopathy is determined based on MRI images. Table 2 shows the long-lasting serum lactate levels rise in line with increasing degrees of HIE. In the group of patients with normal head MRI images, the median serum lactate level was $1.30 \mathrm{mmol} / \mathrm{L}$, while HIE grade I showed a median value of $1.50 \mathrm{mmol} / \mathrm{L}$. In the group of patients with HIE Grade II images,the median value was $3.10 \mathrm{mmol} / \mathrm{L}$, while the median for HIE Grade III was $3.5 \mathrm{mmol} / \mathrm{L}$. The analysis showed that there were differences in serum lactate levels at each degree of HIE based on brain MRI $(p=0.021)$. However, there were no significant differences between serum lactate level and outcomes in SE children $(p=0.187)$, as shown in Table 3.

Table 2 Analysis of long-lasting serum lactate levels with HIE grading on MRI in SE children

Long-lasting Serum Lactate Level

\begin{tabular}{llllllll}
\hline HIE Grading & N & Mean & SD & Median & Min & Max & $\mathbf{P}$ \\
\hline Normal & 2 & 1.30 & - & 1.30 & 1.30 & 1.30 & \\
\hline HIE grade I & 20 & 1.69 & 0.94 & 1.50 & 0.70 & 3.20 & $0.021^{*}$ \\
\hline HIE grade II & 12 & 3.32 & 1.08 & 3.10 & 2.20 & 5.30 & \\
\hline HIE grade III & 8 & 3.48 & 0.78 & 3.50 & 2.70 & 4.20 &
\end{tabular}

*significant at $a=0.05$ (Kruskal-Wallis test) 
Table 3 Analysis of long-lasting serum lactate levels with outcome in SE children

Blood Lactate Level

\begin{tabular}{llllllll}
\hline Outcomes & N & Mean & SD & Median & Min & Max & P \\
\hline Live without neurological deficit & 12 & 1.98 & 0.99 & 1.80 & 0.70 & 3.20 & \\
\hline Live with neurological deficit & 28 & 2.49 & 1.15 & 2.70 & 0.80 & 4.20 & $0.187^{\star}$ \\
\hline Death & 2 & 5.30 & - & 5.30 & 5.30 & 5.30 &
\end{tabular}

*significant at $\mathrm{a}=0.05$ (Kruskal-Wallis test)

We evaluated the association between serum lactate levels, the degree of HIE and patient outcomes. The results of the Spearman analysis showed a significant correlation between serum lactate levels with the degree of $H I E(p=0.001, r s=0.674)$. There was also a significant correlation between HIE grading and patient outcomes $(p=0.004, r s=0.594)$.

\section{Discussion}

Several studies researched the importance of imaging, especially in SE. In brain imaging studies, individuals with SE will initially experience swelling of the brain, and brain volume will gradually decrease afterwards. The inner grey matter in the

brain was the most susceptible structure to damage from prolonged seizures. ${ }^{16}$ Structural and functional damage in several regions of the brain with decreased consciousness due to seizures can be found on MRI of the head with DTI sequences. Some of the regions that are often affected by function and structure are the thalamus, brainstem, and pathways of consciousness. In one study, the Apparent Diffusion Coefficient (ADC) value was higher $(p<0.05)$ in the bilateral dorsal thalamus and postero-superior midbrain in patients with seizures. Therefore, it can be stated that there is a disturbance of the thalamus and upper brain stem, which is part of

the brain that plays an important role as an indicator of seizure patients with impaired consciousness. ${ }^{17}$ DTI is a modern imaging modality that be able to determine the movement of water called Brownian motion. It can detect the neuronal microstructure and other deformity that are not discovered by conventional MRI. It also has the advantage of delineating the microstructure of central nervous system during brain growth and maturation. ${ }^{17}$

This study carried out a head MRI examination with contrast with sequence

DTI to visualize neuronal damage caused by SE seizures, followed by MR spectroscopy to see lactate markers in the brain. Almost all SE children had abnormalities on head MRI. Patients with HIE Grade 1 was the most neuroimaging pattern obtained in this study. Gunawan reported a similar result for their MRI findings in SE children. The picture of MRI abnormalities obtained is HIE Grade 1 as high as $41.7 \%$, HIE Grade 2 as much as $33.3 \%$, and HIE degree 3 in $25 \%$ of children. ${ }^{18}$ 
Lactate levels in the blood will increase significantly in the first 60 minutes after brain damage. There is opinion that increased lactate levels are transient, when the seizures, lactate production will reduce and disappears quickly. ${ }^{10}$ Serum lactate concentration may decreased over time. In a mild brain injury, the serum lactate level will remain low and in severe injury, it will decreased over time. ${ }^{14}$ This study found an increase in long-lasting ( 24 hours measurement following SE) serum lactate levels (>1.3 mmol/L). This occurred due to the increase in anaerobic glucose metabolism during hypoxic events that occurred in tonic-clonic seizures. ${ }^{11,12}$ When the blood barrier in central nervous system is open due to an inflammation, whether originated by infection, seizures related hypoxic lesions, or traumatic brain injury, there will be an increase in lactate levels which can be evaluated from the blood. This characteristic makes lactate a significant clinical marker in detecting brain cell impairment that observed during seizures. ${ }^{17}$

Research to determine the association between serum long-lasting lactate levels and neuroimaging is still limited. This study aims to determine whether the increase in lactate levels in long period in the blood of children with SE is related to brain imaging in terms of detecting functional brain damage. According to the results, the serum lactate levels rise in line with increasing degrees of HIE. This study showed a correlation between serum long-lasting lactate levels with the degree of HIE based on a brain MRI. Blood flow to the brain increases in the early seizure phase and decreases in the later stages as blood pressure decreases. At the same time, brain metabolic rates for glucose and oxygenation persist during SE. Lactate accumulation and ATP depletion are related with hypermetabolic neuronal necrosis. The excitotoxicity mechanism conciliated by the glutamate N-Methyl-D-Aspartate (NMDA) as well as nonNMDA receptors open ion channels, increasing calcium permeability. This contributes to neuronal damage in SE. ${ }^{19}$ One of the biomarkers with increased levels of lactate can provide sensitive results as a predictor of brain damage. A study that conducted on neonates found an evidence of elevated serum lactate 72 hours post-HIE with hypothermia therapy. The neonates that demonstrated abnormal brain MRI findings were related with poor neurological outcome. ${ }^{8}$

A previous study stated that lactate was a prognostic factor in the incidence of SE, where the higher the blood lactate level, the worse the outcome. ${ }^{19}$ Meanwhile, the correlation between the degree of encephalopathy based on MRI of the head and the outcome in children with SE showed a significant relationship. The appearance of changes in MRI of the head reflects a pattern of brain damage (predominant basal ganglia in "acute- total", a predominant watershed in "prolonged-partial" or "severeglobal" damage) and correlates strongly with neurodevelopmental abnormalities. ${ }^{20}$

The limitation of the study is that long-lasting serum lactate levels can also be affected by extracranial disorders. Therefore, further research is needed to compare lactate levels in the blood with cerebrospinal fluid so that lactate levels can be determined which are not affected by the patient's extracranial conditions or other underlying disease.

\section{Conclusion}


In conclusion, it was shown that long-lasting serum lactate level was associated with abnormalities of brain MRI. Besides, it was demonstrated that HIE Grade I was the most common brain damage pattern in SE patients. Moreover, there is a correlation between brain MRI abnormalities and outcome in children with SE. These results are supposed to be useful in order to provide appropriate management and predict the prognosis of patients who experience SE.

\section{List Of Abbreviation}

$\begin{array}{ll}\text { ADC } & \text { Apparent Diffusion Coefficient } \\ \text { CRP } & \text { C-Reactive Protein } \\ \text { CT } & \text { Computed Tomography } \\ \text { DTI } & \text { Diffusion Tensor Imaging } \\ \text { HIE } & \text { Hypoxic Ischemic Encephalopathy } \\ \text { MRI } & \text { Magnetic Resonance Imaging } \\ \text { NMDA } & \text { N-Methyl-D-Aspartate } \\ \text { SE } & \text { Status Epilepticus }\end{array}$

\section{Declarations}

\section{ACCORDANCE}

Our research involving human data (serum lactate level, MRI and patients data from medical record) and have been approved by the institutional ethics committee.

Name of the ethic committee (Komite Etik Penelitian Kesehatan RSUD Dr. Soetomo Surabaya / Medical Research Ethical Committee Dr. Soetomo General Academic Hospital, Surabaya

Ethical clearance no: 246/Panke.KKE/IV/2017

Ethics approval: Ethical clearance no 246/Panke.KKE/IV/2017granted from Medical Research Ethical Committee Dr. Soetomo General Academic Hospital Surabaya.

Consent for publication: Not applicable

Availability of data and material: The datasets generated and/or analysed during the current study are not publicly available due to institution policy, but are available from the corresponding author on reasonable request. 
Competing interests: The authors declare that they have no competing interests.

Funding: No funding

Author's contributions: Gunawan design conceptual framework, data collection, analysis and interpretation, discussion and summary. Noviandi collected data, revised the manuscript and supervised. Samosir revised the manuscript, figures and tables editing and literature review. All authors discussed the results and to the final version of the manuscript.

\section{Acknowledgement:}

The authors wish to thank the Director of Dr. Soetomo General Academic Hospital, Surabaya, Indonesia and Faculty of Medicine Universitas Airlangga for the ethical approval and endless supports.

\section{References}

1. Smith DM, McGinnis EL, Walleigh DJ, et al.:. Management of status epilepticus in children. J Clin Med. 2016;47:1-19.

2. Glauser T, Shinnar S, Gloss D, et al.: Evidence-based guideline: treatment of convulsive status epilepticus in children and adults: report of the Guideline Committee of the American Epilepsy Society. Epilepsy Curr. 2016;<background-color:\#FFCC66;bvertical-align:super;>16</backgroundcolor:\#FFCC66;bvertical-align:super;>(1):48-61.

3. Moghaddasi M, Joodat R, Ataei E. Evaluation of short-term mortality of status epilepticus and its risk factors. J of Epilepsy Res. 2014;5:1-4.

4. Pujar SS, Neville BG, Scott RC, et al.: Death within 8 years after childhood convulsive status epilepticus: a population-based study. Brain. 2011;134:2819-27

5. Barzegar M, Shiva S, Banaeian GR. Etiology and short-term outcome of children with convulsive status epilepticus admitted to tabriz children hospital, Iran. J Anal Res Clin Med. 2014;2:112-7.

6. Kumar M, Kumari R, Narain NG. Clinical profile of status epilepticus (SE) in children in a tertiary care hospital in bihar. J Clin Diagn Res. 2014;8:14-7.

7. Vestergaard MB, Lindberg U, Aachmann-Andersen $\mathrm{NJ}$, et al:: Acute hypoxia increase the cerebral metabolic rate- a magnetic resonance imaging study. J Cereb Blood Flow Metab. 2015;1:1-13.

8. Chiang MC, Lien R, Chu SH, et al:: Serum lactate, brain magnetic resonance imaging and outcome of neonatal hypoxic ischemic encephalopathy after therapeutic hypothermia. Pediatr Neonatol. 2016;57:35-40.

9. Pratamastuti D, Gunawan PI, Saharso D. Serum neuron specific enolase is increased in pediatric acute encephalitis syndrome. Korean J Pediatr. 2017;60(9):302-6.

10. Andersen LW, Mackenhauer J, Roberts JC, et al:: Etiology and therapeutic approach to elevated lactate. Mayo Clin Proc. 2013;88:1127-40. 
11. Nass RD, Zur B, Elger CE, Holdenrieder S, et al:: Acute metabolic effects of tonic-clonic seizures. Epilepsia open. 2019;4:599-608.

12. Matz $\mathrm{O}$, Zdebik $\mathrm{C}$, Zechbauer $\mathrm{S}$, et al.: Lactate as a diagnostic marker in transient loss of consciousness. Seizure. 2016;40:71-5

13. Calabrese VP, Gruemer HD, James K, et al.: Cerebrospinal fluid lactate levels and prognosis in status epilepticus. Epilepsia. 1991;32:816-21.

14. Wu TWW, Tamrazi B, Hsu KH, et al.: Cerebral lactate concentration in neonatal hypoxic-ischemic encephalopathy: in relation to time, characteristic of injury, and serum lactate concentration. Front Neurol. 2018;9:293.

15. Trivedi R, Rathore RK, Gupta RK. Review: Clinical application of diffusion tensor imaging. Indian J Radiol Imaging. 2008;18:45-52.

16. Tsuchida TN, Barkovich AJ, Bollen AW, Hart AP, Ferriero DM. Childhood status epilepticus and excitotoxic neuronal injury. Pediatr Neurol 2007;36:253-7

17. Xie F, Xing W, Wang X, et al:: Altered states of consciousness in epilepsy: A DTI study of the brain. Int J Neurosci. 2016;127:667-72.

18. Gunawan PI, Saharso D, Sari DP. Correlation of serum S100B levels with brain magnetic resonance imaging abnormalities in children with status epilepticus. Korean J Pediatr. 2019;62(7):281-5.

19. Wasterlain CG, Fujikawa DG, Penix L, et al.:Pathophysiological mechanisms of brain damage from status epilepticus. Epilepsia. 1993;34:37-53.

20. Martinello K, Hart AR, Yap S, et al.: Management and Investigation of neonatal encephalopathy: 2017 update. Arch Dis Child Fetal Neonatal Ed. 2017;4:346-58.

\section{Figures}



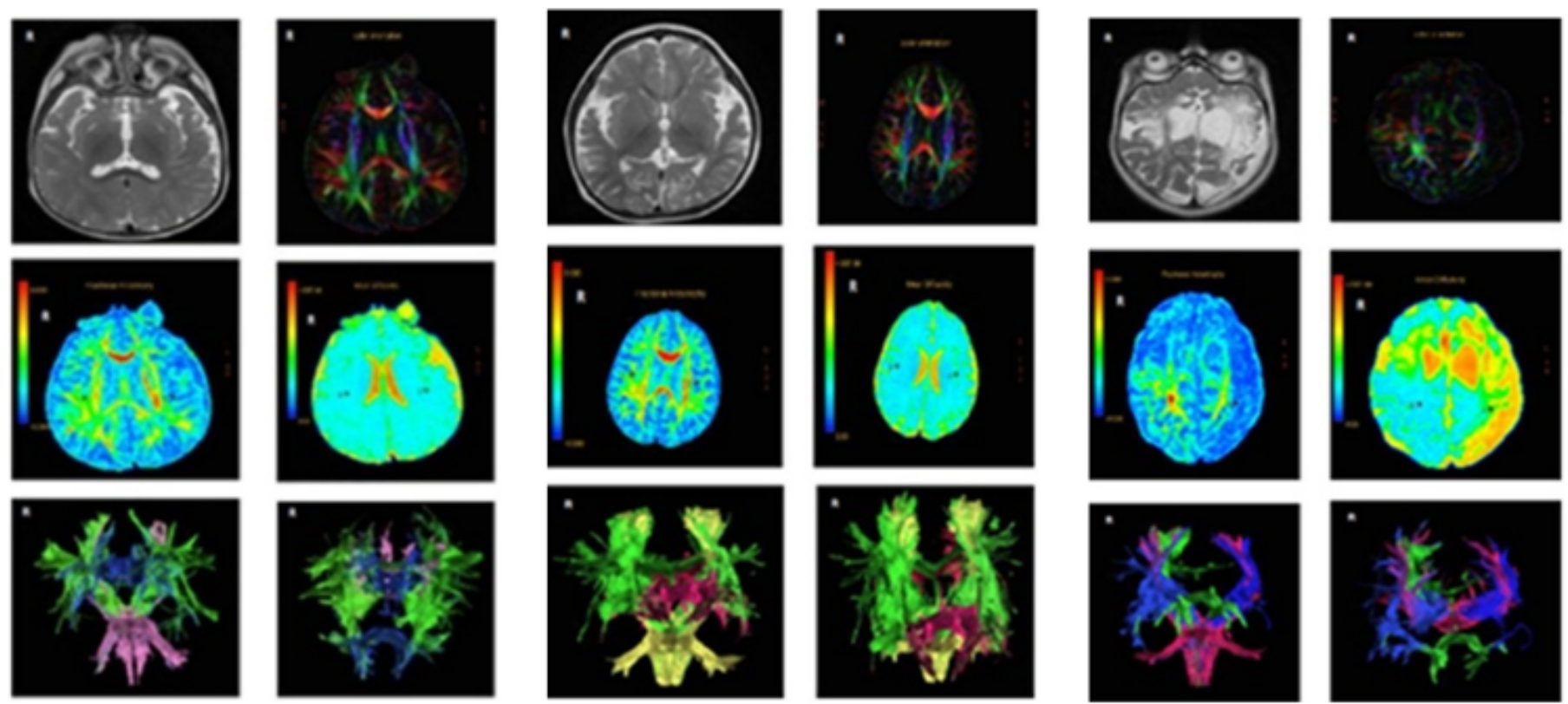

a

$\mathrm{b}$
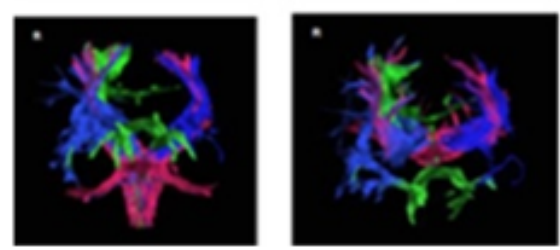

$\mathrm{C}$

Figure 1

Visualized brain MRI of the patient with SE; a. HIE grade I; b. HIE grade Il; c. HIE grade III. 